\title{
Increase in CGRP- and nNOS-immunoreactive neurons in the rat trigeminal ganglion after infusion of an NO donor
}

\author{
Anne Dieterle, Michael JM Fischer, Andrea S Link, Winfried \\ L Neuhuber and Karl Messlinger
}

\begin{abstract}
Background: Nitrovasodilators, such as glyceroltrinitrate (GTN), which produce nitric oxide (NO) in the organism, are known to cause delayed headaches in migraineurs, accompanied by increased plasma levels of calcitonin gene-related peptide (CGRP) in the cranial venous outflow. Increases in plasma CGRP and NO metabolites have also been found in spontaneous migraine attacks. In a rat model of meningeal nociception, infusion of NO donors induced activity of neurons in the spinal trigeminal nucleus.

Methods: Isoflurane-anaesthetised rats were intravenously infused with GTN $(250 \mu \mathrm{g} / \mathrm{kg})$ or saline for two hours and fixed by perfusion after a further four hours. Cryosections of dissected trigeminal ganglia were immunostained for detection of CGRP and neuronal NO synthase (nNOS). The ganglion neurons showing immunofluorescence for either of these proteins were counted.

Results: The proportions of CGRP- and nNOS- as well as double-immunopositive neurons were increased after GTN infusion compared to saline treatment in all parts of the trigeminal ganglion (CGRP) or restricted to the ophthalmic region (nNOS). The size of immunopositive neurons was not significantly different compared to controls.

Conclusion: High levels of NO may induce the expression or availability of CGRP and nNOS. Similar changes may be involved in nitrovasodilator-induced and spontaneous headache attacks in migraineurs.
\end{abstract}

\section{Keywords}

Calcitonin gene-related peptide, nitric oxide, immunohistochemistry, headache

Date received: 26 January 2010; revised: 7 April 2010; accepted: 7 April 2010

\section{Introduction}

Various clinical studies in humans (1-3) and experimental observations in animals (4-6) have shown that nitric oxide (NO) may play an important role in the pathophysiology of primary headaches. Intravenous application of the NO donor glyceroltrinitrate (GTN, $2.5-20 \mu \mathrm{g} / \mathrm{kg}$ over $20 \mathrm{~min}$ ) to healthy subjects provoked headaches, which could be prevented by sumatriptan $6 \mathrm{mg}$ given subcutaneously (SC) (7). Comparing healthy subjects with patients suffering from migraine with aura, GTN infusion $(0.5 \mu \mathrm{g} / \mathrm{kg}$ over $20 \mathrm{~min})$ caused immediate headaches, which were more severe in headache patients (1). Headaches gradually decreased in the control subjects, while they peaked in headache patients five to eight hours later and fulfilled migraine criteria in half of the patient group.

In a rat model of meningeal nociception, infusion of the NO donors sodium nitroprusside (SNP, $25 \mu \mathrm{g} / \mathrm{kg}$ ) or GTN $(250 \mu \mathrm{g} / \mathrm{kg})$ increased the spontaneous neuronal activity of neurons in the spinal trigeminal nucleus (STN) with meningeal afferent input $(4,8)$. This increase in activity was biphasic in that an immediate weak activation was followed by a second phase of activity slowly developing within one to two hours (4), and it could be reversed by infusion of the CGRP receptor antagonist BIBN4096BS (8). Thus the comparably high dose of NO donor may have converted a normal rat into an animal model reflecting some similarities to migraine. The pathophysiological processes behind

University of Erlangen-Nuernberg, Germany.

\section{Corresponding author:}

Karl Messlinger, Institute of Physiology and Pathophysiology, University of Erlangen-Nuernberg, Universitaetsstrasse 17, D-91054 Erlangen, Germany

Email: messlinger@physiologiel.uni-erlangen.de 
these findings are not known. However, regarding the delayed onset of migraine-like headaches, NO might be able to trigger migraine attacks by initiating changes in gene expression. Endogenous NO is synthesised by three distinct isoforms of NO synthase (NOS): endothelial NOS (eNOS), neuronal NOS (nNOS) and inducible NOS (iNOS) (9). The nNOS isoenzyme is widely distributed in the mammalian central nervous system and has been detected in the trigeminal ganglion as well $(10,11)$. As the inhibition of endogenous NO synthesis in migraineurs has been reported to attenuate migraine attacks (12) and to decrease the spontaneous spinal trigeminal activity in rats (4), a tonic NO effect on the mediation of migraine attacks has been suggested. This theory was supported by clinical observations demonstrating an increase of NO metabolites such as nitrites and nitrates in internal jugular vein blood of migraineurs during spontaneous migraine attacks (13). Thus, NO may play a role in both initiating and mediating migraine attacks.

Calcitonin gene-related peptide (CGRP) is one of the most common neuropeptides in the central and peripheral nervous systems (14). In migraineurs clinical studies have revealed increased plasma levels of CGRP during both spontaneous attacks and nitrovasodilator-induced headaches $(2,13,15)$. Moreover, recent clinical trials have shown that inhibition of CGRP receptors with the CGRP-receptor antagonists olcegepant (BIBN4096BS) and telcagepant (MK-0974) is effective in the acute treatment of migraine attacks $(16,17)$. In addition, BIBN4096BS reduced NO-induced increases in neuronal activity in the STN in the mentioned rat model of meningeal nociception (8). As both CGRP and NO are involved in spontaneous and induced migraine headaches, there may be a link between NO infusion and expression of CGRPand NO-producing enzymes. An immunohistological analysis of the trigeminal ganglion demonstrated coexpression of CGRP and NOS in the majority of NOS-immunopositive neurons (10). Moreover, various in vitro experiments have shown that $\mathrm{NO}$ stimulation causes CGRP release from the spinal cord, meninges and trigeminal ganglion cells (18-20). Bellamy et al. (21) demonstrated in primary cultures of rat trigeminal neurons that NO donors are even able to increase transcription of the CGRP gene by promoter activation. However, these findings have not yet been confirmed in vivo.

The aim of this study was to investigate whether NO donors increase the expression of CGRP and nNOS in neurons within the rat trigeminal ganglion. Thus, after infusion of GTN, the number of CGRP-, nNOS-immunoreactive and double immunopositive trigeminal neurons was analysed and compared to those of saline-treated animals.

\section{Material and methods}

\section{Animals}

Twelve adult male inbred Wistar rats (290-310 g) were used for the study. Animals were housed at a 12 hour: 12 hour light-dark cycle with ad libitum access to food and water. All procedures were conducted in accordance with principles of Laboratory Animal Care (National Institutes of Health [USA] publication no. 86-23, revised 1985), the European Communities Council Directive and German regulations of animal welfare and treatment. Experimental protocols were approved by the local government.

\section{Treatment, perfusion, fixation, preparation and tissue processing}

Rats were initially anaesthetised in a closed box by inhalation of $5 \%$ isoflurane (Abbott, Wiesbaden, Germany). During surgery animals breathed oxygenenriched (30\%) and humidified air with $2.5 \%$ isoflurane supplied by a loosely fitted mask. A catheter was inserted into the femoral vein for application of substances. Isoflurane was reduced to $1.5 \%$ and the animals were placed in a prone position on a feedback-controlled warming plate in order to maintain body temperature. Six animals were slowly intravenously (IV) infused with the NO donor glyceroltrinitrate (GTN, Schwarz Pharma, Monheim, Germany) at a dose of $250 \mu \mathrm{g} / \mathrm{kg}$ over a period of two hours. After testing different infusion rates and doses of GTN in previous experiments, the infusion parameters were adjusted so that no significant lowering of the arterial pressure occurred (8). Six control animals received isotonic saline with an identical infusion rate and duration. All substances were diluted in isotonic saline and infused by a syringe pump (Harvard Apparatus, March-Hugstetten, Germany). After infusion all animals were under anaesthesia for a further four hours. Then the concentration of isoflurane in the inspiration gas was increased to $5 \%$, the animals were quickly thoracotomised and perfused through the left ventricle with isotonic saline at room temperature for about two minutes, followed by a solution of $4 \%$ paraformaldehyde in $10 \mathrm{mM}$ phosphate-buffered saline (PBS, pH 7.4) for 20 minutes. After an extended craniectomy and cervical laminectomy, the supratentorial dura mater was dissected from the brain. Cerebrum and brainstem were removed and the trigeminal ganglia excised from the skull base. Trigeminal ganglia were postfixed by immersion in $4 \%$ paraformaldehyde for two hours and stored in $10 \mathrm{mM}$ PBS for 24 hours. For cryoprotection the ganglia were incubated in a $30 \%$ solution of sucrose in PBS for one day. 
After mounting on Tissue-Tek (GSV1, Slee Technik, Mainz, Germany), the ganglia were transferred to a plastic micro test tube, rapidly frozen at $-70^{\circ} \mathrm{C}$ by immersing the tube in methylbutane cooled in liquid nitrogen and stored in the freezer at $-20^{\circ} \mathrm{C}$. A series of $20-\mu \mathrm{m}$ thick longitudinal sections were cut from the ganglia with a cryostat (Leica, Bensheim, Germany). Sections were mounted on poly-L-lysine-coated slides and dried for one hour at room temperature prior to staining.

\section{Double-labelling immunohistochemistry}

The sources, characteristics and dilutions of the primary and secondary antibodies used in this study are listed in Tables 1 and 2. For immunostaining the mounted sections were rinsed in a solution of PBS containing antimicrobial sodium azide $\left(\mathrm{NaN}_{3}\right)$ and preincubated with a solution of $5 \%$ normal donkey serum (Dako, Hamburg, Germany) containing 0.5\% Triton $\mathrm{X}-100$ and $1 \%$ bovine serum albumine (BSA) (both from Sigma-Aldrich, Steinheim, Germany) in PBS/ $\mathrm{NaN}_{3}$ for one hour at room temperature. After rinsing in $\mathrm{PBS} / \mathrm{NaN}_{3}$ for five minutes, the sections were incubated with primary antibodies directed against nNOS and CGRP at room temperature overnight. All primary antibodies were diluted in $\mathrm{PBS} / \mathrm{NaN}_{3}$, containing $1 \%$ BSA and $0.5 \%$ Triton X-100. Sections were washed with $\mathrm{PBS} / \mathrm{NaN}_{3}$ three times for 5 minutes and incubated with donkey anti-rabbit immunoglobulin $\mathrm{G}$ (IgG), coupled to Alexa 488 for detection of nNOS, and donkey anti-goat $\mathrm{IgG}$, coupled to Alexa 555 for detection of CGRP, for one hour at room temperature. All secondary antibodies were diluted in PBS with $\mathrm{NaN}_{3}$ containing $1 \%$ BSA and $0.5 \%$ Triton X-100. Sections were rinsed with $\mathrm{PBS} / \mathrm{NaN}_{3}$ three times, coverslipped in PBS-glycerol $(\mathrm{pH}$ 8.6) and stored in the refrigerator where they were prevented from light.

Specificity of the immunocytochemical reactions was verified by sections incubated in solutions lacking primary antibodies. No specific staining was found with ALEXA Fluor 488 (green) or with ALEXA Fluor 555 (red) but in the green channel all neurons showed a faint background staining, which was used to count the whole cell number. The pattern of CGRP-immunoreactivity (-ir) was identical to previous descriptions (22-24). Enzyme-linked immunosorbent assay (ELISA) was used to evaluate the anti-CGRP primary antibody (manufacturer's technical information). The rabbit anti-nNOS antibody has been used in various rat preparations $(25,26)$ and its specificity was tested by preabsorption with an excess of the relevant antigen (27).

\section{Azur methylene blue staining}

Four overview micrographs representative sections of the trigeminal ganglion were stained with an azur methylene blue solution containing $1 \mathrm{~g} / 1$ azur II (Merck, Germany; catalogue \# 1.09211) and $1 \mathrm{~g} / 1$ methylene blue (Merck, Germany; \# 1283) in distilled water with $1 \%$ sodium tetraborate. The slides with the mounted sections were rinsed for 10 minutes in distilled water, then placed on a heating plate at $50^{\circ} \mathrm{C}$, stained with drops of the solution for 30 seconds and rinsed again in distilled water, before they were embedded in Kaiser's glycerine and coverslipped.

\section{Light microscopy and cell counting}

The quantitative analysis of the sections was performed using a Leica Aristoplan epifluorescence microscope (Leica, Bensheim, Germany) equipped with an HBO 100 W-2 lamp (Osram, Munich, Germany), connected

Table I. Primary antibodies used for immunohistochemistry

\begin{tabular}{|c|c|c|c|}
\hline Name & Host & Source & Dilution \\
\hline Calcitonin gene-related peptide (CGRP) & Goat & Biotrend, Cologne, Germany; BT I7-2090-07 & $1: 100$ \\
\hline Neuronal nitrogenmonooxyde synthase (nNOS) & Rabbit & Dr. Mayer, University of Graz & $1: 800$ \\
\hline
\end{tabular}

Table 2. Secondary antibodies used for detection of primary antibodies

\begin{tabular}{llll}
\hline Secondary antibodies & Conjugated to & \multicolumn{1}{c}{ Source } & Dilution \\
\hline Donkey anti-rabbit & ALEXA Fluor & Molecular Probes, Eugene, OR, USA; & \\
& 488 & A2I206 I: I000 \\
Donkey anti-goat & ALEXA Fluor & Molecular Probes, Eugene, OR, USA; \\
& 555 & A2I432 & \\
\hline
\end{tabular}


to a CCT camera (Spot RT, Visitron Systems, Puchheim, Germany). For detecting fluorescence we used filter block N2.1 for red emission (ALEXA Fluor 555) and filter block I3 for green emission (ALEXA Fluor 488).

In each ganglion the 34 central sections of the whole series were selected, from which every second section was analysed in order to avoid double counting; that is, 17 sections per ganglion were analysed. Using a 40x dry objective lens, neurons with clearly visible nuclei were counted. CGRP- and nNOS-immunoreactive (-ir) cells as well as double-stained cells were analysed. As a reference, the total cell count of all neurons visible in the background of the green channel was used. Neurons located in the medial region (ophthalmic, V1) of the trigeminal ganglion and in the lateral regions (mandibular, V3, and maxillary, V2) were separately counted (see Figure 1C). The investigator counting the neurons (AD) was blinded to the experiments from which the sections were derived.

\section{Confocal microscopy and image processing}

In addition to the quantitative light microscopic analysis, images were obtained using a BioRad MRC 1000 confocal laser scanning system (Bio-Rad, Hemel
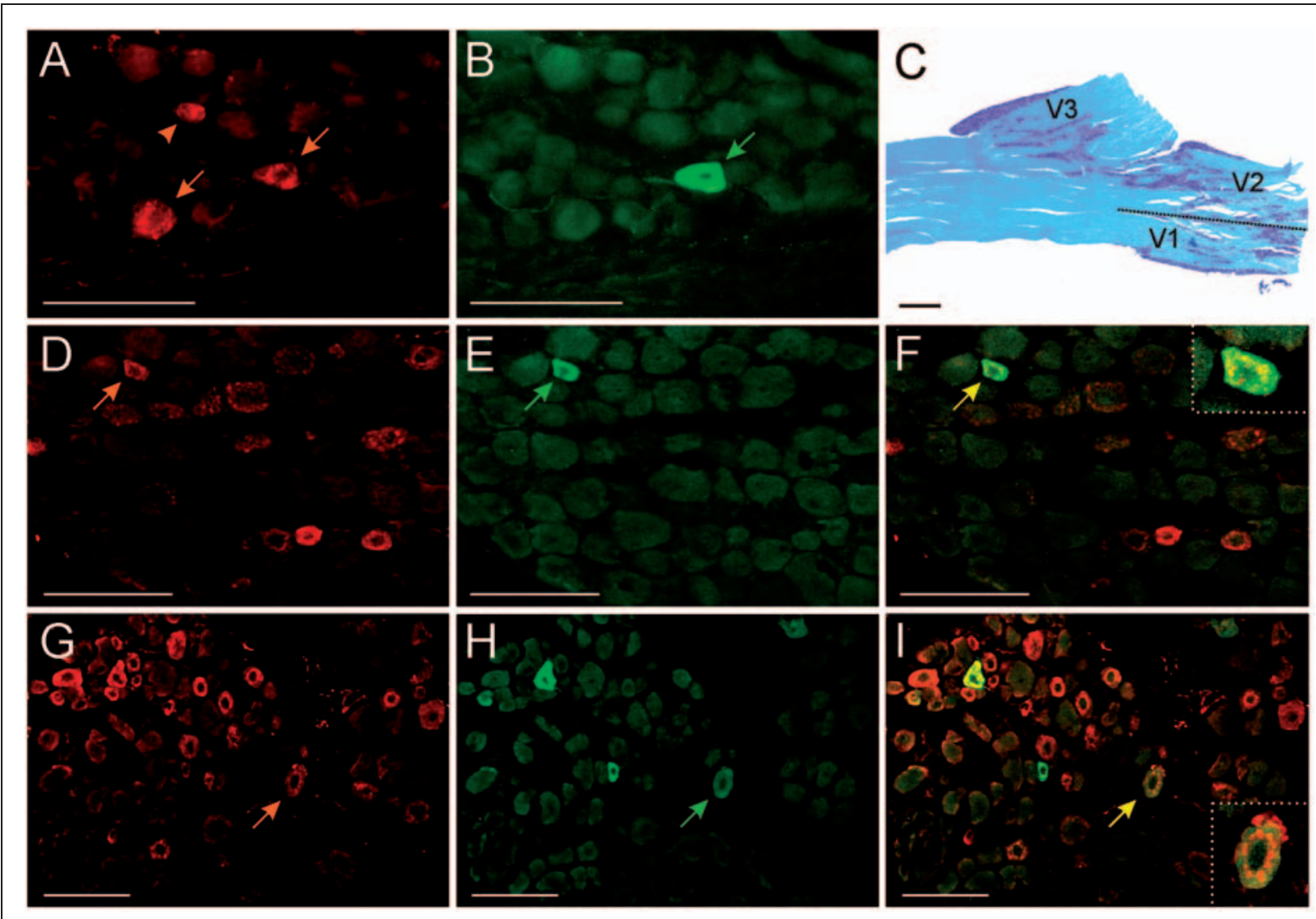

Figure I. CGRP and nNOS immunofluorescence in the trigeminal ganglion. (A, B) Light microscope images showing neurons in the maxillar region immunopositive for CGRP ( $\mathrm{A}$, red arrows) and $\mathrm{nNOS}$ ( $\mathrm{B}$, green arrow) as visualised for cell countings. CGRP- and nNOS-ir are co-localised in one neuron. The cell marked by an arrowhead is not counted in this section, because it lacks a visible nucleus. (C) Low-power micrograph of a trigeminal ganglion section stained with azur methylene blue showing nerve fibre plexus (blue) and neuronal cell bodies (purple) in the three trigeminal partitions, VI-V3. Neurons were counted separately in the VI half of the VI/2 region, as indicated by a broken line. (D-F) Confocal images of the ophthalmic region of a trigeminal ganglion after saline pretreatment. CGRP (D) and nNOS immunofluorescence $(E)$ is co-localised in one cell (arrow), visible in the merged image $(F)$ as yellow granules within the neuron (inset at higher magnification). (G-l) Confocal images of the ophthalmic region of a trigeminal ganglion after GTN pretreatment. One relatively large CGRP- and nNOS-positive neuron is visible in the upper left, one small exclusively nNOS-positive neuron in the middle. In another neuron (arrow), weak CGRP $(\mathrm{G})$ and nNOS immunofluorescence $(\mathrm{H})$ is seen but not strictly co-localised, visible as red granules (CGRP-ir) on a green background (nNOS-ir) within the cell in the merged image (l, inset at higher magnification). Size bars $100 \mu \mathrm{m}$, panel C $500 \mu \mathrm{m}$. CGRP = calcitonin gene-related peptide. $\mathrm{nNOS}=$ neuronal nitric oxide synthase. $\mathrm{nNOS}-\mathrm{ir}=\mathrm{nNOS}$ immunoreactivity. GTN $=$ glycerol trinitrate. 
Hempstead, UK) equipped with a three line kryptonargon laser (American Laser Technology, Salt Lake City, UT, USA) and attached to a Nikon Diaphot 300 microscope (Nikon, Düsseldorf, Germany). The filter settings of the BioRad confocal scanner for double label were: 488-nm excitation for ALEXA 488 (filter 522 DF32), 568-nm excitation for ALEXA 555 (filter 605 DF322). A 20x dry objective lens (numerical aperture 0.75 ) was used. Electronic zoom factors varied between 1.0 and 3.6.

Images of $768 \times 512$ pixels were obtained, and the channels were merged into a 24-bit red-green-blue (RGB) tif file by using confocal assistant software 4.02. To test for co-localisation, single optical sections at the same focus plane were taken and the two corresponding channels were merged. Only adjustment for contrast, brightness and evenness of illumination was performed. To apply text and scale bars and organise the final layouts, CorelDraw (Corel, Dublin, Ireland) was used.

\section{Statistical analysis}

The number of CGRP-, nNOS- as well as co-localised cells was compared between GTN and saline-treated animals. Further, the number of nNOS- and CGRPpositive cells in medial and lateral regions of the trigeminal ganglion were analysed. Statististical analysis was performed with STATISTICA (Tulsa, OK, USA). The number of neurons depended on the plane of sections with the maximum of cells counted in the central slices $(\mathrm{R}=0.33, \quad p=.001 \quad$ in saline-treated animals). Therefore, the number of cells in corresponding slices from animals pretreated with saline and GTN were compared as matched pairs. T-test for dependent samples was used for $n \geq 10$, the Wilcoxon rank test for smaller samples and analysis of variance (ANOVA) for three or more groups. Significance was accepted for $p<.05$. The data are reported as mean \pm standard error of the mean (SEM).

\section{Results}

In the selected sections of the trigeminal ganglion, the total number of neurons and the number of neurons showing CGRP-, nNOS-immunoreactivity or co-localisation of both markers were separately quantified using light microscopy (Figures 1A and 1B). In the first countings the number of nNOS-immunoreactive neurons seemed to be especially high in the medial (ophthalmic) region (V1) of the trigeminal ganglion. Therefore, in three animals per group the immunoreactivity was separately counted in the V1 region and in the mandibulo-maxillary division (V2/3, lateral) of the trigeminal ganglion (Figure 1C). Confocal imaging was used to visualise the intracellular distribution of immunoreactivity and to take representative micrographs (Figure 1D-I).

As a reference, the total cell count was detected by counting all neurons visible in the background of the green channel. A similar number of cells was counted in the groups treated by saline and GTN $(p=0.25$; Figure 2D).

\section{CGRP immunoreactive neurons}

CGRP-ir neurons were either completely stained or marked by granular immunofluorescent structures around the nucleus (Figures 1A, 1D and 1G). Cells containing this marker were widely distributed throughout the trigeminal ganglion. The average number of CGRP-positive neurons in the saline-treated animals was $53 \pm 5$ per slice, in the GTN-treated animals $93 \pm 4(p=.028$; Figure 2A). The increase in CGRP-immunopositive cells after GTN treatment was found both in V1 and in V2/3 (ANOVA $\mathrm{F}_{(1,8)}=6.4, p=.035$, HSD post-hoc tests both $p<.001$; Figure 3 ). The size of CGRP-ir and nNOS-ir neurons was not significantly different between GTN-treated and control animals.

\section{nNOS immunoreactive neurons}

Cells immunoreactive for nNOS mostly showed a homogeneous green staining (Figures 1B, 1E and 1H). The average number of nNOS-ir neurons per slice was $3.6 \pm 0.8$ in the saline-treated animals, and $8.0 \pm 0.8$ in the GTN-treated group ( $p=.028$, Figure 2B). After infusion of GTN an increase in the number of nNOS positive neurons was observed in the $\mathrm{V} 1$ division (ANOVA $\mathrm{F}_{(1,8)}=7.7, p=.024$, HSD (honestly significant difference) post-hoc test $p=.004$ ), but not in the V2/3 region $(p=.61$, HSD post-hoc test; Figure 3$)$.

\section{Double immunopositive cells}

Co-localised neurons were identified by a CGRPimmunopositive staining in the red channel and an nNOS-immunopositive staining in the green channel. In the merged confocal images the double positive neurons appeared homogeneously or partly yellow (Figure 1F, I). They were rarely found throughout the trigeminal ganglion. On average $1.0 \pm 0.3$ cells per slice in the saline-treated and $2.5 \pm 0.5$ in GTN-treated animals were detected $(p=.028$, Figure $2 \mathrm{C}$ ).

\section{Discussion}

In this study we found that pretreatment with the NO donor GTN increased the number of trigeminal ganglion neurons immunoreactive to CGRP as well as the number of nNOS-immunopositive and 


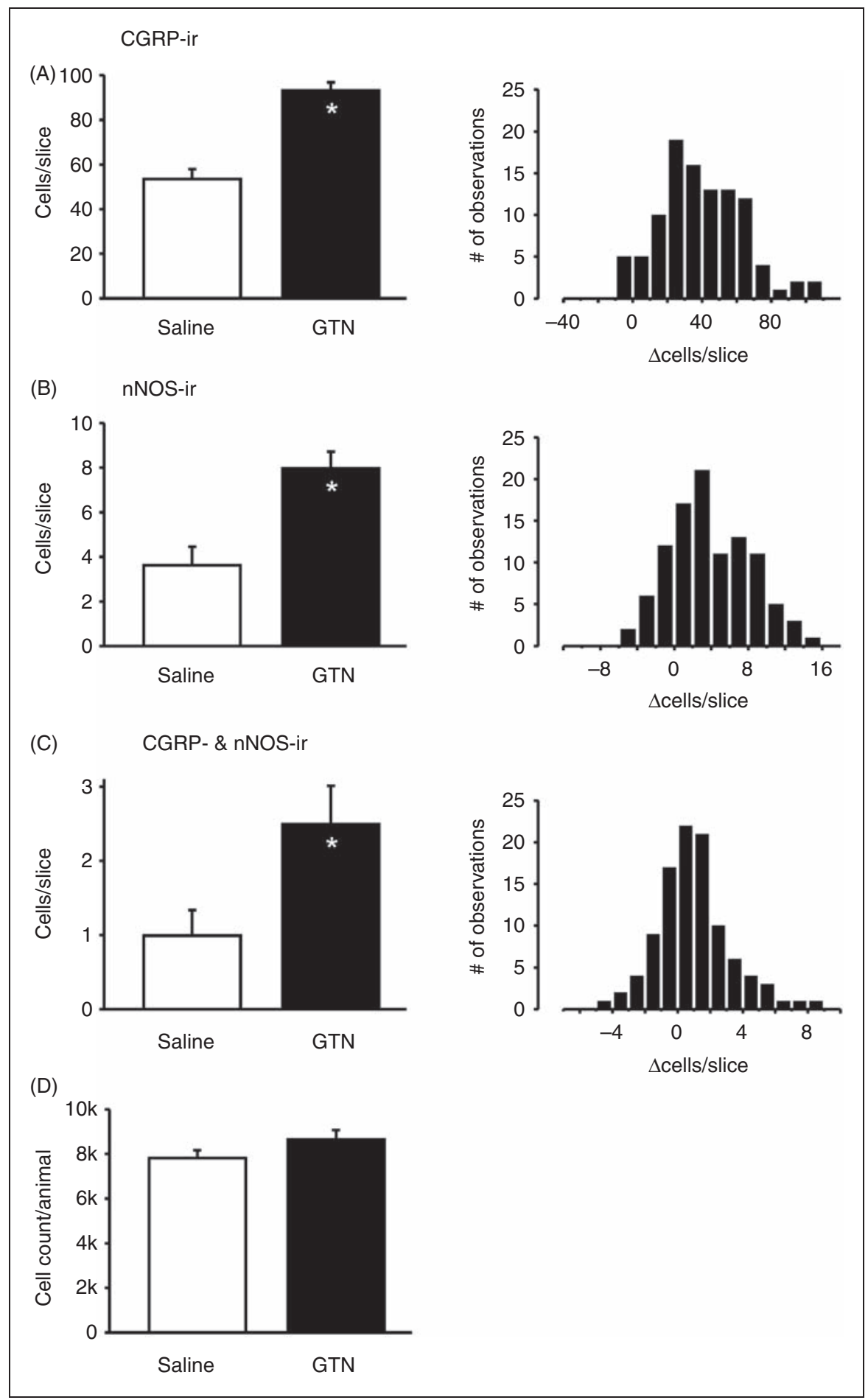

Figure 2. Number of immunoreactive cells for CGRP (A), nNOS (B) and both (C) in animals infused with either saline or GTN. The left panels show the mean \pm SEM, the right panels are histograms (number of observations) of the difference $(\Delta$, GTN minus salinetreated animals) in cells per slice of corresponding sections. (D) Total cell count (in thousands) from saline- and GTN-treated animals. $\mathrm{CGRP}=$ calcitonin gene-related peptide. $\mathrm{nNOS}=$ neuronal nitric oxide synthase. $\mathrm{SEM}=$ standard error of the mean. $\mathrm{GTN}=$ glycerol trinitrate. 


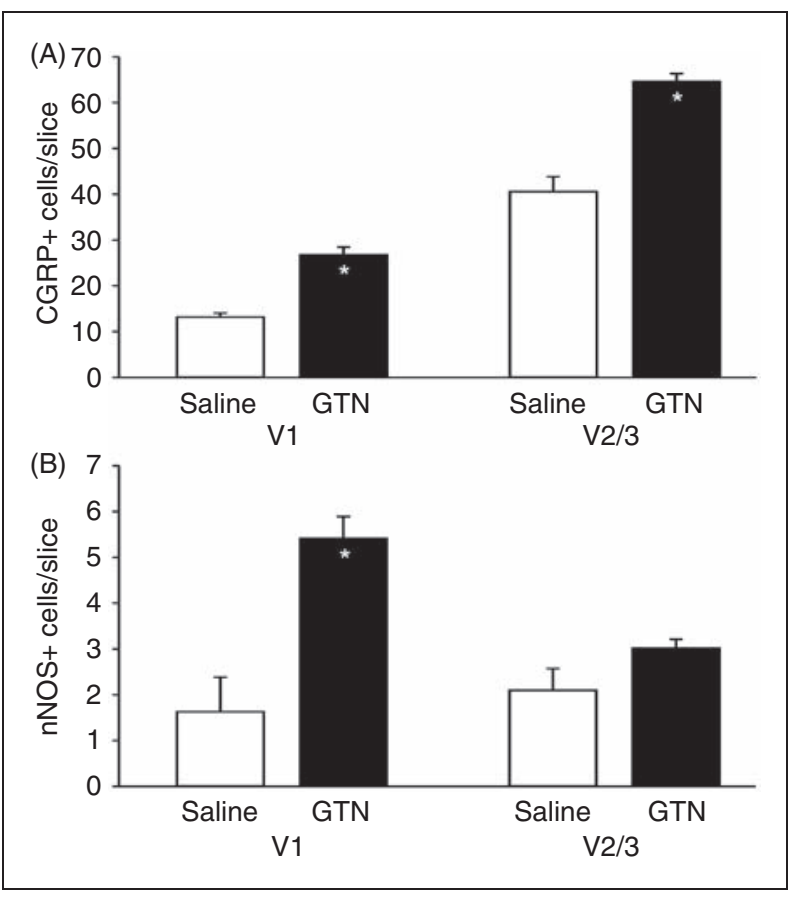

Figure 3. Number of CGRP- (A) and nNOS- (B) immunopositive neurons in the medial $(\mathrm{VI})$ and lateral (V2/3) divisions of the trigeminal ganglion. CGRP = calcitonin gene-related peptide. $\mathrm{nNOS}=$ neuronal nitric oxide synthase .

double-immunopositive cells. Furthermore, nNOSpositive neurons were particularly increased in the ophthalmic region of the trigeminal ganglion. This may be functionally important, as the ophthalmic division is considered to be the most frequently affected region in migraine, although animal studies suggest that all divisions of the trigeminal ganglion receive afferent projections from the dura (28). Our results suggest that NO produced by GTN is able to induce the expression of both CGRP and nNOS in neurons previously immunonegative or below the immunohistochemical detection level for these proteins. Alternatively, it may be assumed that the immunoreactivity of CGRP and nNOS was increased by changes in intracellular storing or the molecular structure of these proteins such as nitrosylation induced by the GTN infusion (see below).

\section{Structural and functional effects caused by NO donors}

In a recent electrophysiological study in the rat, in which we recorded from neurons in the spinal trigeminal nucleus caudalis with afferent input from the cranial dura mater, the same dose of GTN as used in the present study $(250 \mu \mathrm{g} / \mathrm{kg} \mathrm{IV})$ as well as the NO donor sodium nitroprusside (SNP, $25 \mu \mathrm{g} / \mathrm{kg}$ IV) increased the ongoing neuronal activity within one hour (8), confirming the results of an older study with SNP infusion (4). To see if these functional changes are associated with structural changes, we examined histochemical markers for NOS isoforms in the spinal trigeminal nucleus after infusion of SNP $(50 \mu \mathrm{g} / \mathrm{kg})$ and found that the number of NADPH-diaphorase-positive neurons increased continuously from three to eight hours after SNP infusion and the number of nNOS-immunoreactive neurons increased significantly four hours after SNP infusion (29). This was in line with reports about an increase in nNOS levels in the spinal trigeminal nucleus caudalis four hours after subcutaneous injection of $10 \mathrm{mg} / \mathrm{kg}$ GTN (6,30). In addition, we recognised an increase in the density of NADPH-diaphorase- and nNOS-positive nerve fibres in the spinal trigeminal tract, assuming that $\mathrm{nNOS}$ in primary trigeminal afferents may also be upregulated after NO donor infusion. This initiated the present study to look at the trigeminal ganglion four hours after the SNP infusion, and we determined not only nNOS- but also CGRP-immunoreactive neurons, as it was reported that NO donors can increase the CGRP expression in trigeminal ganglion cell cultures (21).

\section{Regulation of gene expression of CGRP and $n N O S$}

CGRP is expressed by alternative splicing of the primary gene product of CALCA and in addition by CALCB, resulting in two CGRP isoforms, alpha- and betaCGRP, the first of which is predominant in neurons (31). It has previously been demonstrated that the alpha-CGRP gene is regulated by intracellular signalling cascades, in particular by mitogen-activated protein kinase (MAPK) pathways (32,33). Bellamy et al. (21) have shown in an in vitro model of primary cultures of rat trigeminal neurons that superfusion of the $\mathrm{NO}$ donor S-nitroso-N-acetylpenicillamine (SNAP) causes an increase in the release of CGRP concomitant with an activation of the CGRP gene promoter. Using immunocytochemistry they found that this treatment also increased the number of neurons that contain JNK and p38 in their nuclei, suggesting that these two MAPKs are involved in the induction of CGRP expression by NO donors. The present study suggests that similar processes are induced by NO donors in vivo confirming changes in this trigeminal cell culture system (21), which could have been influenced by the culture conditions.

The transcription of nNOS in cultivated cortical neurons has been shown to be dependent on calcium influx and the cAMP response element-binding (CREB) transcription factor (34). The expression of nNOS in human neuroblastoma cells is activated by acetylation of the nuclear factor kappa B (NF-kappaB) (35). Activation of this factor is also involved in $\mathrm{nNOS}$ expression preceding the 
development of experimental hyperalgesia (36). NO induces calcium influx, possibly by membrane depolarisation or cGMP-dependent calcium channels (37), activates multiple protein kinases in various tissues (38) and together with calcium promotes activation of CREB in neuronally derived cell lines (39). Thus, NO could stimulate the expression of nNOS, and the intracellular machinery for a NO donor to induce nNOS may be present also in trigeminal ganglion neurons.

\section{Alternative regulation of CGRP and nNOS immunoreactivity}

Messenger RNA for neuronal and endothelial (but not inducible) NOS has been identified in rat trigeminal ganglia not subjected to culture (40). Thus, CGRP and nNOS production can be expected to be regulated on the transcriptional as well as translational level. However, in a series of recent experiments two or six hours after pretreatment of rats with GTN, we found no evidence for an upregulation of CGRP mRNA in the trigeminal ganglion using real-time polymerase chain reaction (PCR) or of total CGRP protein using an ELISA. The same treatment caused an increase in CGRP release from the isolated ganglion stimulated by inflammatory mediators (41). Thus, the present data could also reflect subcellular changes in the distribution of CGRP, which may modify the access or binding of antibodies. The CGRP immunofluorescence appeared in different forms, either as conspicuous granular structures usually surrounding the nucleus, or as homogeneous staining of the whole cell (see Figures 1A, 1D and $1 G)$. The granular structures are reminiscent of intracellular stores such as endoplasmic reticulum or Golgi apparatus. Neuropeptides such as CGRP are thought to be stored in, transported by and released from large vesicles with or without dense cores (42-45). Thus, an alternative explanation for the increase in numbers of immunoreactive neurons may be an opening of these intracellular stores caused by infusion of the NO donor, so that more neurons gain sufficient immunoreactivity to be detected by immunofluorescence.

Apart from the most common intracellular mechanism of NO via activation of guanylate cyclase and increase in cGMP, other direct reactions of NO have been described, such as nitration and nitrosylation (reviewed in 46). Thus, it could also be speculated that nitrated or nitrosylated CGRP and nNOS are more immunoreactive to the respective antibodies.

\section{Possible role of NO and CGRP in meningeal nociception and headache}

Although it is unclear which process underlies the described changes in immunoreactivity of trigeminal ganglion neurons, they are accompanied by dramatic functional changes of the intracranial trigeminal system. Infusion of NO donors causes increased activity and responsiveness to meningeal stimulation of spinal trigeminal neurons in animals $(4,8)$ and induces delayed migraine-like attacks in migraineurs (1). This was concomitant with an increase in plasma CGRP levels suggesting enhanced CGRP release (2), which was absent in healthy volunteers after infusion of GTN (47). We have recently found that infusion of an NO donor causes also delayed upregulation of putatively NO-producing neurons in the spinal trigeminal nucleus (29). An increase in NO production after infusion of NO donors has not yet directly been shown, but elevated plasma levels of NO metabolites and increased exhaled NO gas have been found in spontaneous migraine attacks (13) and migraine-like headaches induced by NO donor administration $(48,49)$. NO is known as a "retrograde transmitter" to facilitate spinal transmitter and neuropeptide release and enhance synaptic transmission throughout the central nervous system (50-52). CGRP may also enhance spinal release of excitatory neurotransmitters (53) from spinal terminals of primary afferents, which have been shown to carry abundant CGRP receptors $(24,54)$. CGRP released from spinal terminals of primary afferents is thought to facilitate nociceptive transmission (55) and may contribute to the increase in spinal trigeminal activity, as inhibition of CGRP receptors blocks this activity (56). Taken these findings together, we suggest in this study that the enhanced CGRP and nNOS immunoreactivity of trigeminal ganglion neurons may reflect the delayed functional changes towards an increased nociceptive activity obviously induced by $\mathrm{NO}$ in animal experiments as well as in migraine patients.

\section{Implications for vascular versus neuronal hypotheses of migraine pain}

GTN was infused at a very low rate to avoid vasodilatation and lowering of the blood pressure, which could have influenced the metabolism in the trigeminal ganglion. Nevertheless, the question may arise as to whether direct vascular effects of NO in the trigeminal ganglion or trigeminal tissues may have been supportive for the changes observed after GTN infusion, as NO and CGRP are potent vasodilators of intra- and extracranial arteries involved in neurogenic processes $(10,57-59)$. This issue is interesting with regard to the vascular and neuronal hypotheses of migraine.

The original vascular hypothesis of migraine, which held that vascular motility was responsible for migraine pain, was later modified and extended to the neurogenic inflammation hypothesis (reviewed in 60). The model of meningeal neurogenic inflammation in rodents, which 
was valuable in predicting the effectiveness of antimigraine drugs (61), includes the dilatation of meningeal blood vessels through neuropeptides released from trigeminal afferents and mediators, such as histamine released from degranulated mast cells $(62,63)$. An increase in CGRP-releasing and nNOS-producing trigeminal neurons could in principle facilitate neurogenic vasodilatation in the meninges, provided that these peptides are carried by axonal transport to the peripheral terminals, where CGRP and NO can be released (64). However, this process probably does not occur rapidly enough to explain the induction of migraine attacks within few hours after NO donor infusion (1).

Another question is if the infused NO donors have similar effects in the cranial dura mater as observed in the trigeminal ganglion, that is, if they induce an increase in NOS producing cells and changes in CGRP-releasing nerve fibres. NO donors, though at much higher doses than used in the present study, released CGRP from the rat cranial dura, promoting increases in meningeal blood flow (20). In a rat model of intravital microscopy, neurogenic vasodilatation of meningeal blood vessels was attenuated by inhibition of nNOS, while CGRP-induced vasodilatation was attenuated by eNOS inhibition (65). Specific inhibition of iNOS was not effective in this model. Meningeal vasodilatation caused by CGRP, however, does not excite trigeminal afferents innervating the rat cranial dura mater (66). Therefore, it is unlikely that vascular effects in the cranial dura induced by NO or CGRP cause nociceptive inputs to the trigeminal ganglion, which can be responsible for the increase in CGRP- and nNOS-ir neurons. We assume, rather, that there is a direct impact of the NO donor on trigeminal ganglion cells, which is also evidenced by the expression experiments performed on cultured trigeminal ganglion neurons (21). These data, if transferred to humans, are supportive for a neuronal rather than a vascular hypothesis of migraine generation.

Clinical trials have shown that unspecific inhibition of NO synthases can relieve migraine pain (67) and decrease tension-type headache (68). With regard to the above preclinical findings and the present study, it seems likely that it is the inhibition of nNOS and eNOS, rather than iNOS, which causes this therapeutic effect. Specific iNOS inhibitors such as GW274150, which has been shown to be analgesic in rat models of inflammatory and neuropathic pain (69), may thus be less suited to treat migraine pain. Using immunohistochemistry, we have seen co-localisation of the CGRPreceptor proteins CLR and RAMP1 indicating functional CGRP receptors in the rat trigeminal ganglion on a large subgroup of neurons (24), and another subgroup of neurons showed immunoreactivity for soluble guanylate cyclase (sGC), the intracellular receptor for
NO (unpublished results). An intraganglionic, possibly reciprocal, signalling between nNOS and CGRPexpressing neurons could therefore play a role in trigeminal nociception. The NO production within the ganglion may build up and increase the activity of spinal trigeminal neurons (4), a process likely to cause headaches in patients suffering from primary headaches (70).

\section{Acknowlegements}

We acknowledge Karin Löschner, Birgit Vogler, Jana Schramm and Maria Schulte for their excellent technical assistance. The present study was upported by the BMBF (German Headache Consortium).

\section{References}

1. Christiansen I, Thomsen LL, Daugaard D, Ulrich V and Olesen J. Glyceryl trinitrate induces attacks of migraine without aura in sufferers of migraine with aura. Cephalalgia 1999; 19: 660-667.

2. Juhasz G, Zsombok T, Modos EA, et al. NO-induced migraine attack: strong increase in plasma calcitonin gene-related peptide (CGRP) concentration and negative correlation with platelet serotonin release. Pain 2003; 106 : 461-470.

3. Olesen J, Thomsen LL, Lassen LH and Olesen IJ. The nitric oxide hypothesis of migraine and other vascular headaches. Cephalalgia 1995; 15: 94-100.

4. Koulchitsky S, Fischer MJ, De Col R, Schlechtweg PM and Messlinger K. Biphasic response to nitric oxide of spinal trigeminal neurons with meningeal input in rat-possible implications for the pathophysiology of headaches. J Neurophysiol 2004; 92: 1320-1328.

5. De Col R, Koulchitsky SV and Messlinger KB. Nitric oxide synthase inhibition lowers activity of neurons with meningeal input in the rat spinal trigeminal nucleus. Neuroreport 2003; 14: 229-232.

6. Pardutz A, Szatmari E, Vecsei L and Schoenen J. Nitroglycerin-induced nNOS increase in rat trigeminal nucleus caudalis is inhibited by systemic administration of lysine acetylsalicylate but not of sumatriptan. Cephalalgia 2004; 24: 439-445.

7. Iversen HK and Olesen J. Headache induced by a nitric oxide donor (nitroglycerin) responds to sumatriptan. A human model for development of migraine drugs. Cephalalgia 1996; 16: 412-418.

8. Koulchitsky S, Fischer M and Messlinger K. Calcitonin gene-related peptide receptor inhibition reduces neuronal activity induced by prolonged increase in nitric oxide in the rat spinal trigeminal nucleus. Cephalalgia 2008; 29: 408-417.

9. Knowles RG and Moncada S. Nitric oxide synthases in mammals. Biochem J 1994; 298(Pt 2): 249-258.

10. Edvinsson L, Mulder H, Goadsby PJ and Uddman R. Calcitonin gene-related peptide and nitric oxide in the trigeminal ganglion: cerebral vasodilatation from trigeminal nerve stimulation involves mainly calcitonin generelated peptide. J Auton Nerv Syst 1998; 70: 15-22. 
11. Lazarov N and Dandov A. Distribution of NADPH-diaphorase and nitric oxide synthase in the trigeminal ganglion and mesencephalic trigeminal nucleus of the cat. A histochemical and immunohistochemical study. Acta Anat (Basel) 1998; 163: 191-200.

12. Lassen LH, Christiansen I, Iversen HK, Jansen-Olesen I and Olesen $\mathbf{J}$. The effect of nitric oxide synthase inhibition on histamine induced headache and arterial dilatation in migraineurs. Cephalalgia 2003; 23: 877-886.

13. Sarchielli P, Alberti A, Codini M, Floridi A and Gallai V. Nitric oxide metabolites, prostaglandins and trigeminal vasoactive peptides in internal jugular vein blood during spontaneous migraine attacks. Cephalalgia 2000; 20: 907-918.

14. van Rossum D, Hanisch UK and Quirion R. Neuroanatomical localization, pharmacological characterization and functions of CGRP, related peptides and their receptors. Neurosci Biobehav Rev 1997; 21: 649-678.

15. Goadsby PJ, Edvinsson L and Ekman R. Vasoactive peptide release in the extracerebral circulation of humans during migraine headache. Ann Neurol 1990; 28: 183-187.

16. Ho TW, Mannix LK, Fan X, et al. Randomized controlled trial of an oral CGRP receptor antagonist, MK-0974, in acute treatment of migraine. Neurology 2008; 70: 1304-1312.

17. Olesen J, Diener HC, Husstedt IW, et al. Calcitonin generelated peptide receptor antagonist BIBN $4096 \mathrm{BS}$ for the acute treatment of migraine. N Engl J Med 2004; 350: 1104-1110.

18. Garry MG, Walton LP and Davis MA. Capsaicin-evoked release of immunoreactive calcitonin gene-related peptide from the spinal cord is mediated by nitric oxide but not by cyclic GMP. Brain Res 2000; 861: 208-219.

19. Geppetti P, Del Bianco E, Santicioli P, Lippe IT, Maggi CA and Sicuteri F. Release of sensory neuropeptides from dural venous sinuses of guinea pig. Brain Res 1990; 510: 58-62.

20. Strecker T, Dux $M$ and Messlinger $K$. Nitric oxide releases calcitonin-gene-related peptide from rat dura mater encephali promoting increases in meningeal blood flow. J Vasc Res 2002; 39: 489-496.

21. Bellamy J, Bowen EJ, Russo AF and Durham PL. Nitric oxide regulation of calcitonin gene-related peptide gene expression in rat trigeminal ganglia neurons. Eur $J$ Neurosci 2006; 23: 205-266.

22. Wank M and Neuhuber WL. Local differences in vagal afferent innervation of the rat esophagus are reflected by neurochemical differences at the level of the sensory ganglia and by different brainstem projections. J Comp Neurol 2001; 435: 41-59.

23. Raab M and Neuhuber WL. Vesicular glutamate transporter 2 immunoreactivity in putative vagal mechanosensor terminals of mouse and rat esophagus: indication of a local effector function? Cell Tissue Res 2003; 312: 141-148.

24. Lennerz JK, Ruhle V, Ceppa EP, et al. Calcitonin receptor-like receptor (CLR), receptor activity-modifying protein 1 (RAMP1), and calcitonin gene-related peptide (CGRP) immunoreactivity in the rat trigeminovascular system: differences between peripheral and central
CGRP receptor distribution. J Comp Neurol 2008; 507: 1277-1299.

25. Ewald P, Neuhuber WL and Raab M. Vesicular glutamate transporter 1 immunoreactivity in extrinsic and intrinsic innervation of the rat esophagus. Histochem Cell Biol 2006; 125: 377-395.

26. Worl J, Mayer B and Neuhuber WL. Nitrergic innervation of the rat esophagus: focus on motor endplates. $J$ Auton Nerv Syst 1994; 49: 227-233.

27. Bergua A, Schrodl F and Neuhuber WL. Vasoactive intestinal and calcitonin gene-related peptides, tyrosine hydroxylase and nitrergic markers in the innervation of the rat central retinal artery. Exp Eye Res 2003; 77: 367-374.

28. Steiger $\mathrm{HJ}$ and Meakin CJ. The meningeal representation in the trigeminal ganglion - an experimental study in the cat. Headache 1984; 24: 305-309.

29. Schlechtweg PM, Roder J, Fischer MJ, Neuhuber W and Messlinger $\mathrm{K}$. Increase in NADPH-diaphorase-positive and neuronal NO synthase immunoreactive neurons in the rat spinal trigeminal nucleus following infusion of a $\mathrm{NO}$ donor-evidence for a feed-forward process in $\mathrm{NO}$ production involved in trigeminal nociception. Cephalalgia 2009; 29: 566-579.

30. Pardutz A, Krizbai I, Multon S, Vecsei L and Schoenen J. Systemic nitroglycerin increases nNOS levels in rat trigeminal nucleus caudalis. Neuroreport 2000; 11: 3071-3075.

31. Rezaeian AH, Isokane $\mathrm{T}$, Nishibori $\mathrm{M}$, et al. alphaCGRP and betaCGRP transcript amount in mouse tissues of various developmental stages and their tissue expression sites. Brain Dev 2009; 31: 682-693.

32. Durham PL and Russo AF. Stimulation of the calcitonin gene-related peptide enhancer by mitogen-activated protein kinases and repression by an antimigraine drug in trigeminal ganglia neurons. J Neurosci 2003; 23: 807-815.

33. Vause CV and Durham PL. CGRP stimulation of iNOS and NO release from trigeminal ganglion glial cells involves mitogen-activated protein kinase pathways. $J$ Neurochem 2009; 110: 811-821.

34. Sasaki M, Gonzalez-Zulueta $M$, Huang $H$, et al. Dynamic regulation of neuronal NO synthase transcription by calcium influx through a CREB family transcription factor-dependent mechanism. Proc Natl Acad Sci USA 2000; 97: 8617-8622.

35. Li Y, Zhao Y, Li G, et al. Regulation of neuronal nitric oxide synthase exon if gene expression by nuclear factorkappaB acetylation in human neuroblastoma cells. J Neurochem 2007; 101: 1194-1204.

36. Chan CF, Sun WZ, Lin JK and Lin-Shiau SY. Activation of transcription factors of nuclear factor kappa B, activator protein-1 and octamer factors in hyperalgesia. Eur J Pharmacol 2000; 402: 61-68.

37. Ohkuma S and Katsura M. Nitric oxide and peroxynitrite as factors to stimulate neurotransmitter release in the CNS. Prog Neurobiol 2001; 64: 97-108.

38. Schindler $\mathrm{H}$ and Bogdan $\mathrm{C}$. NO as a signaling molecule: effects on kinases. Int Immunopharmacol 2001; 1: $1443-1455$. 
39. Peunova N and Enikolopov G. Amplification of calciuminduced gene transcription by nitric oxide in neuronal cells. Nature 1993; 364: 450-453.

40. Jansen-Olesen I, Zhou M, Zinck T, Xu CB and Edvinsson L. Expression of Inducible Nitric Oxide Synthase in Trigeminal Ganglion Cells during Culture. Basic Clin Pharmacol Toxicol 2005; 97: 355-363.

41. Eberhardt M, Neeb L, Vogel EM, et al. Glyceroltrinitrate facilitates stimulated CGRP release but not gene expression of CGRP or its receptor components in rat trigeminal ganglia. Neuropeptides 2009; 43: 483-489.

42. Buldyrev I, Tanner NM, Hsieh HY, Dodd EG, Nguyen LT and Balkowiec A. Calcitonin gene-related peptide enhances release of native brain-derived neurotrophic factor from trigeminal ganglion neurons. J Neurochem 2006; 99: 1338-1350.

43. Gulbenkian S, Merighi A, Wharton J, Varndell IM and Polak JM. Ultrastructural evidence for the coexistence of calcitonin gene-related peptide and substance $\mathrm{P}$ in secretory vesicles of peripheral nerves in the guinea pig. J Neurocytol 1986; 15: 535-542.

44. Hiura A, Nasu F and Ishizuka H. Relationship of substance P- and CGRP-immunoreactive central endings of the primary afferent neurons to GABAergic interneurons in the guinea pig substantia gelatinosa. Okajimas Folia Anat Jpn 1998; 74: 231-235.

45. Meng J, Wang J, Lawrence G and Dolly JO. Synaptobrevin I mediates exocytosis of CGRP from sensory neurons and inhibition by botulinum toxins reflects their anti-nociceptive potential. $J$ Cell Sci 2007; 120: 2864-2874.

46. Guix FX, Uribesalgo I, Coma M and Munoz FJ. The physiology and pathophysiology of nitric oxide in the brain. Prog Neurobiol 2005; 76: 126-152.

47. Kruuse C, Iversen HK, Jansen-Olesen I, Edvinsson L and Olesen J. Calcitonin gene-related peptide (CGRP) levels during glyceryl trinitrate (GTN)-induced headache in healthy volunteers. Cephalalgia 2010; 30: 467-474.

48. Martelletti P, D'Alo S, Stirparo G, Rinaldi C, Cifone MG and Giacovazzo M. Modulation of nitric oxide synthase by nitric oxide donor compounds in migraine. Int J Clin Lab Res 1998; 28: 135-139.

49. Van der Schueren BJ, Lunnon MW, Laurijssens BE, et al. Does the unfavorable pharmacokinetic and pharmacodynamic profile of the iNOS inhibitor GW273629 lead to inefficacy in acute migraine? J Clin Pharmacol 2009; 49: 281-290

50. Ikeda $\mathrm{H}$ and Murase $\mathrm{K}$. Glial nitric oxide-mediated long-term presynaptic facilitation revealed by optical imaging in rat spinal dorsal horn. J Neurosci 2004; 24: 9888-9896.

51. Vetter G, Geisslinger $G$ and Tegeder I. Release of glutamate, nitric oxide and prostaglandin E2 and metabolic activity in the spinal cord of rats following peripheral nociceptive stimulation. Pain 2001; 92: 213-218.

52. Volgushev M, Balaban P, Chistiakova M and Eysel UT. Retrograde signalling with nitric oxide at neocortical synapses. Eur J Neurosci 2000; 12: 4255-4267.

53. Kangrga I and Randic M. Tachykinins and calcitonin gene-related peptide enhance release of endogenous glutamate and aspartate from the rat spinal dorsal horn slice. J Neurosci 1990; 10: 2026-2038.

54. Marvizon JC, Perez OA, Song B, et al. Calcitonin receptor-like receptor and receptor activity modifying protein 1 in the rat dorsal horn: localization in glutamatergic presynaptic terminals containing opioids and adrenergic alpha2C receptors. Neuroscience 2007; 148: 250-265.

55. Storer RJ, Akerman S and Goadsby PJ. Calcitonin generelated peptide (CGRP) modulates nociceptive trigeminovascular transmission in the cat. Br J Pharmacol 2004; 142: 1171-1181.

56. Fischer MJ, Koulchitsky S and Messlinger K. The nonpeptide calcitonin gene-related peptide receptor antagonist BIBN4096BS lowers the activity of neurons with meningeal input in the rat spinal trigeminal nucleus. $J$ Neurosci 2005; 25: 5877-5883.

57. Escott KJ, Beattie DT, Connor HE and Brain SD. Trigeminal ganglion stimulation increases facial skin blood flow in the rat: a major role for calcitonin gene-related peptide. Brain Res 1995; 669: 93-99.

58. Peitl B, Nemeth J, Szolcsanyi J, Szilvassy Z and Porszasz R. Sensory nitrergic meningeal vasodilatation and nonnitrergic plasma extravasation in anaesthesized rats. Eur J Pharmacol 2004; 497: 293-299.

59. Williamson DJ, Hargreaves RJ, Hill RG and Shepheard SL. Intravital microscope studies on the effects of neurokinin agonists and calcitonin gene-related peptide on dural vessel diameter in the anaesthetized rat. Cephalalgia 1997; 17: 518-524.

60. Moskowitz MA and Macfarlane R. Neurovascular and molecular mechanisms in migraine headaches. Cerebrovasc Brain Metab Rev 1993; 5: 159-177.

61. Buzzi MG and Moskowitz MA. Evidence for 5-HT1B/ $1 \mathrm{D}$ receptors mediating the antimigraine effect of sumatriptan and dihydroergotamine. Cephalalgia 1991; 11: $165-168$.

62. Dimitriadou V, Buzzi MG, Moskowitz MA and Theoharides TC. Trigeminal sensory fiber stimulation induces morphological changes reflecting secretion in rat dura mater mast cells. Neuroscience 1991; 44: 97-112.

63. Williamson DJ, Hargreaves RJ, Hill RG and Shepheard SL. Sumatriptan inhibits neurogenic vasodilation of dural blood vessels in the anaesthetized rat-intravital microscope studies. Cephalalgia 1997; 17: 525-531.

64. Kashihara Y, Sakaguchi M and Kuno M. Axonal transport and distribution of endogenous calcitonin generelated peptide in rat peripheral nerve. J Neurosci 1989; 9: 3796-3802.

65. Akerman S, Williamson DJ, Kaube H and Goadsby PJ. Nitric oxide synthase inhibitors can antagonize neurogenic and calcitonin gene-related peptide induced dilation of dural meningeal vessels. $\mathrm{Br} J$ Pharmacol 2002; 137: 62-68.

66. Levy D, Burstein R and Strassman AM. Calcitonin generelated peptide does not excite or sensitize meningeal nociceptors: implications for the pathophysiology of migraine. Ann Neurol 2005; 58: 698-705.

67. Lassen LH, Ashina M, Christiansen I, et al. Nitric oxide synthase inhibition: a new principle in the treatment of migraine attacks. Cephalalgia 1998; 18: 27-32. 
68. Ashina M, Lassen LH, Bendtsen L, Jensen R and Olesen J. Effect of inhibition of nitric oxide synthase on chronic tension-type headache: a randomised crossover trial. Lancet 1999; 353: 287-289.

69. De Alba J, Clayton NM, Collins SD, Colthup P, Chessell I and Knowles RG. GW274150, a novel and highly selective inhibitor of the inducible isoform of nitric oxide synthase (iNOS), shows analgesic effects in rat models of inflammatory and neuropathic pain. Pain 2006; 120 : 170-181.

70. Olesen J, Thomsen LL, Lassen LH and Olesen IJ. The nitric oxide hypothesis of migraine and other vascular headaches. Cephalalgia 1995; 15: 94-100. 